\title{
Can surface magnetic fields reproduce solar irradiance variations in cycles 22 and $23 ?$
}

\author{
T. Wenzler ${ }^{1}$, S. K. Solanki ${ }^{2}$, and N. A. Krivova ${ }^{2}$ \\ ${ }^{1}$ Institute of Astronomy, ETH Zentrum, 8092 Zurich, Switzerland \\ e-mail: wenzler@astro.phys.ethz.ch \\ 2 Max-Planck-Institut für Sonnensystemforschung ${ }^{\star}, 37191$ Katlenburg-Lindau, Germany \\ e-mail: solanki@linmpi.mpg.de
}

Received 22 June 2004 / Accepted 17 November 2004

\begin{abstract}
Total solar irradiance is reconstructed using data from the spectromagnetograph on Kitt Peak for 1992-2003 (covering parts of cycles 22 and 23) and compared with observational data. The model assumes that solar irradiance variations are caused by surface magnetism and its single free parameter is kept at the same value for both cycles. A good correspondence is found for the whole period of time, with no bias between the two cycles on time-scales longer than the solar rotation period. This is in agreement with the recent results of de Toma et al. (2004), but it is in contrast to the earlier analysis of de Toma et al. (2001), and suggests that the source of the irradiance variations is the same for cycles 22 and 23, namely the evolution of the magnetic flux at the solar surface.
\end{abstract}

Key words. Sun: solar-terrestrial relations - Sun: activity - Sun: magnetic fields

\section{Introduction}

The Sun's total irradiance is known to vary by about $1 \mathrm{~W} \mathrm{~m}^{-2}$ over the solar activity cycle (e.g. Fröhlich 2003) and by larger amounts on shorter time scales. For cycle 23 it has been shown that over $90 \%$ of the irradiance variations are due to the evolution of the surface magnetic field (e.g. Krivova et al. 2003). de Toma et al. (2001) have suggested that cycles 22 and 23 are different in that during its rising phase, cycle 23 shows a similar increase in total solar irradiance (TSI) to cycle 22, although the total magnetic flux and traditional solar activity indices indicate that cycle 23 is weaker than cycle 22 . de Toma et al. (2001) also found that empirical models of TSI based on sunspot deficit and facular/network excess in cycle 22 underestimate the TSI measurements (VIRGO and ACRIM II) in 1999. They interpreted this to imply either a problem in the observations or a change in the source of radiative variability on the Sun. In contrast, de Toma et al. (2004) found that the TSI increase from solar minimum to maximum in cycle 23 agrees with estimates from irradiance indices. It is important to independently model the irradiance in both cycles, since the implications of different sources of irradiance changes in both cycles are far-reaching. For example, the measured irradiance record is used to calibrate reconstructions of longer-term irradiance, which are used as input for climate studies. Also, a basic assumption of such reconstructions is that the irradiance variations of all cycles have the same physical cause.

\footnotetext{
^ Previously Max-Planck-Institut für Aeronomie.
}

Here we extend the work of Krivova et al. (2003) and Wenzler et al. (2004) to test whether the surface magnetic field can simultaneously reproduce the irradiance in cycles 22 and 23, as proposed by de Toma et al. (2004), or cannot, as claimed by de Toma et al. (2001). One advantage of our model is that it describes solar irradiance variations with a single free parameter, while de Toma et al. $(2001,2004)$ make use of two.

\section{Data}

The data we employ is the set of daily full-disk magnetograms and the corresponding continuum intensity images recorded by the spectromagnetograph (SPM; Jones et al. 1992) of the National Solar Observatory (NSO) at the Kitt Peak Vacuum Tower (KPVT). Data recorded by this instrument in the Fe I $8688 \AA$ spectral line are available for 2894 days from 1992 November 21 to 2003 September 21. Earlier magnetograms were obtained either in a different spectral line or with a different instrument. Due to the difficulty of obtaining reliable cross-calibrations, the accuracy of reconstructions based on multiple instruments is lower, so that for the present analysis we consider only the time span mentioned above.

The SPM data (magnetograms and continuum intensity images) occasionally exhibit artifacts introduced by clouds, seeing and/or instrumental problems. We identified regions with artifacts or obvious loss of data quality (often also missing parts of the image, where a scan was not completed) on 839 days, constituting $29 \%$ of the original data record. Thus data for 
2055 days can be used for irradiance reconstructions. A more complete description of the SPM data and the selection procedure is given by Wenzler et al. (2004), who also demonstrated that these data allow high quality reconstructions to be made. Note that 1992 is after the maximum of cycle 22, but Fig. 3 of de Toma et al. (2001) shows that also after 1992 the irradiance in cycle 22 exhibited a sufficiently different behaviour to cycle 23 . Furthermore, in 2003 cycle 23 is at the same phase as cycle 22 was in 1992-1993, so that we can compare the same phases of both cycles.

Total solar irradiance reconstructions based on SPM data are compared with the composite (version 30) of TSI from PMOD/WRC, Davos, Switzerland (Fröhlich 2000; Fröhlich 2003), which for the period considered here is based on VIRGO/SoHO (Fröhlich et al. 1995) and ACRIM II/UARS (Willson 1994) data. We also compare separately with the TSI measurements of the ACRIM II experiment, which was active for most of the considered period.

\section{Model}

We use the same model as Fligge et al. (2000a,b) and Krivova et al. (2003), which is based on the evolution of the solar surface magnetic field. In that model the position and flux density of magnetic features on the solar disk are extracted from full-disk magnetograms. These magnetic features are classified as either sunspot umbrae, sunspot penumbrae or faculae, based on their brightness in full-disk continuum images. Pixels with magnetic flux below a given threshold are classified as quiet Sun, so that 4 atmospheric components are employed for the modelling. Each component is described by a time independent spectrum obtained from a model atmosphere ascribed to that component. The atmospheres are taken from Unruh et al. (1999). The number and location, i.e. the heliocentric angle $\theta$, of the pixels assigned to each component are time dependent. The heliocentric angle $\theta$ of the pixels is important due to the centre-to-limb variation of the brightness contrast of magnetic features. The number of pixels and $\theta$ values are obtained from the magnetograms and continuum images. There is one additional complication: whereas a pixel either belongs to a sunspot umbra or penumbra or it does not, for faculae the unresolved magnetic elements may cover a pixel to a larger or lesser extent. A filling factor, which gives the fractional coverage of the pixel by the facular model, is therefore introduced. With increasing magnetogram signal, $B$, the filling factor and hence the contribution of the facular component to the pixel increases. A limiting value, $B_{\text {sat }}$, is introduced beyond which the pixel is completely filled by faculae (and described purely by the facular atmosphere). Fligge et al. (2000b) called this parameter $\tilde{\Phi}_{3}$. The model is able to accurately reproduce both total and spectral solar irradiance changes over cycle 23, irrespective of whether MDI data (Krivova et al. 2003) or SPM data are used (Wenzler et al. 2004).

The reconstructions presented here differ from those carried out by Wenzler et al. (2004) firstly in the interval over which they extend (1992-2003 instead of 1996-2001) and secondly in that we here use unbinned, i.e. original resolution SPM data and a fixed threshold of $21 \mathrm{G}$ to identify faculae. Since the main aim of Wenzler et al. (2004) was to compare the reconstructed total solar irradiance based on the two data sets, SPM and MDI, respectively, they used the MDI noise threshold throughout and binned the SPM data to the same pixel size as the MDI data.

Most importantly, however, the single free parameter of the model $B_{\text {sat }}$ is now determined so as to best reproduce the TSI data using SPM. We have determined and employed throughout a value of $B_{\mathrm{sat}}=310 \mathrm{G}$ when comparing with the PMOD composite, i.e. this single value was used irrespective of the interval of time over which we compared the reconstruction with the composite irradiance. This leads to a slightly improved correspondence with the VIRGO data. The correlation coefficient $r_{\mathrm{c}}=0.94$, instead of $r_{\mathrm{c}}=0.92$, as found Wenzler et al. (2004), who employed the $B_{\text {sat }}$ value found by (Krivova et al. 2003) using MDI data. We stress, however, that we have also carried out reconstructions using exactly the same model as Wenzler et al. (2004). The conclusions of this paper remain unaffected.

\section{Results}

The reconstructed total solar irradiance for 2055 days between November 1992 and September 2003 based on SPM data is presented in Fig. 1 (filled circles connected by dotted curve). The composite total irradiance measurements compiled by C. Fröhlich (version 30) are given by the solid lines. Daily values are plotted for both observed and reconstructed irradiance. The top panel demonstrates that the longer term trends of the TSI are well reproduced over the full 11 years of the reconstruction, including the descending phase of cycle 22 . The two middle and the two lower panels display extracts on an enlarged scale at different phases of the solar cycles 22 and 23, respectively. These four panels reveal that the short-term variations of the total solar irradiance in both solar cycles, 22 and 23, are also well reproduced. In particular, the enlargements I and IV refer to similar phases of cycles 22 and 23, respectively. The observations are well reproduced during both. Enlargement II lies at a similar activity level as I and IV, but during the rising phase of cycle 23 , and is also well reproduced.

In Fig. 2 the reconstructed value based on SPM data is plotted against the measured irradiance (PMOD composite measurements). The resulting correlation coefficient is $r_{\mathrm{c}}=0.94$. The linear regression to the dots (dashed line) is practically indistinguishable from the expectation value (solid line). de Toma et al. (2004) used a linear regression model with two components related to magnetic regions. Their resulting correlation coefficients of the TSI estimates based on this empirical models vs. TSI measurements (PMOD composite measurements) from 1986 to 2003 are of the order of 0.93, i.e. similar to the value we find. Note that our model has only a single free parameter in contrast to the model of de Toma et al. $(2001,2004)$, which has two free parameters. Also, our model is based on the assumption that solar irradiance changes are entirely caused by the evolution of the solar surface magnetic field.

Figure 3 shows the difference between the reconstructed total solar irradiance and the composite measurements. The dots in the top panel are daily values, the bigger filled circles are averages over 10 days, which show trends more clearly. 

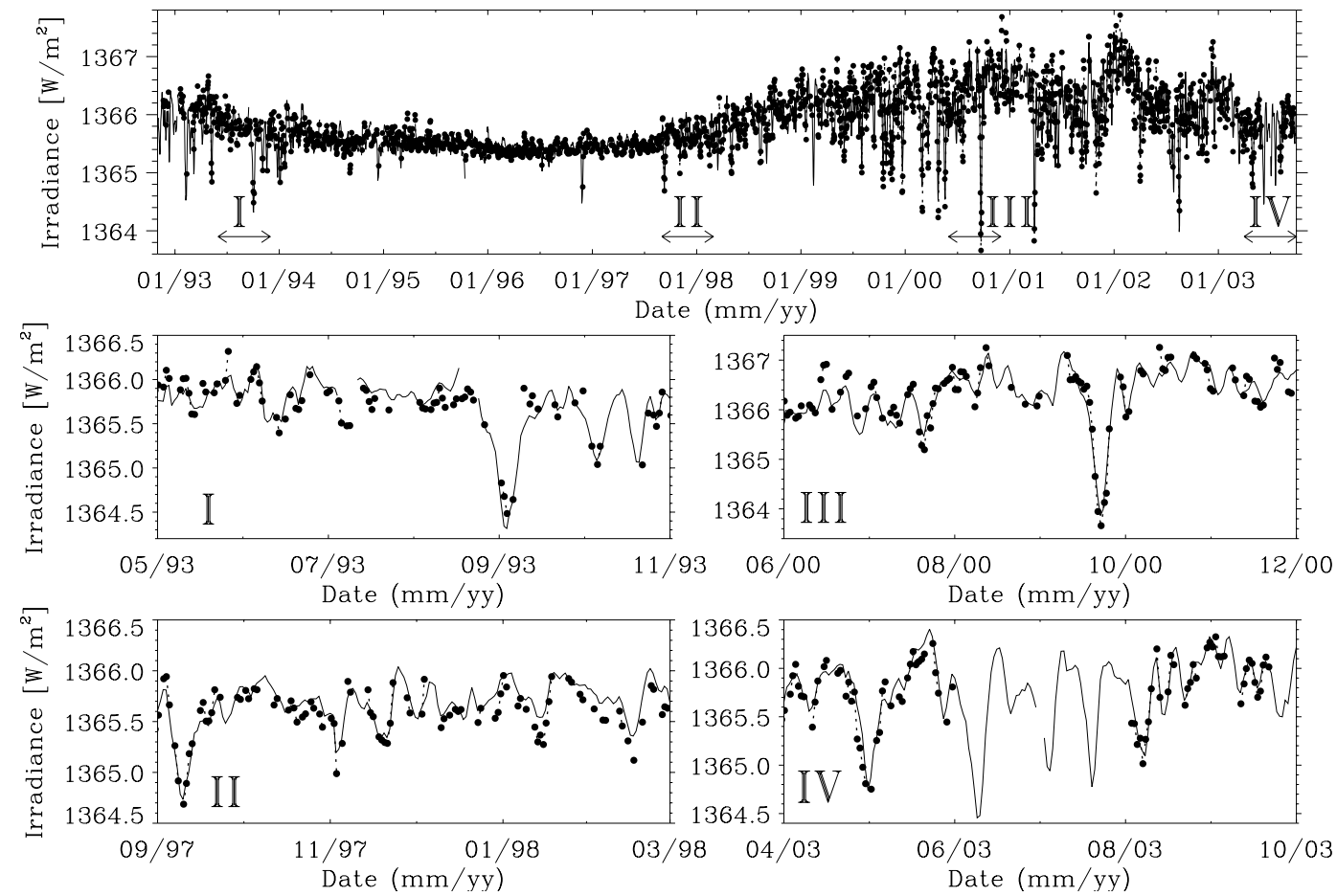

Fig. 1. Composite record of total solar irradiance compiled from detailed cross-calibrations of various daily radiometeric measurements (solid line) and reconstructed daily TSI based on SPM data (filled circles, connected by the dotted curve when there are no data gaps) for 2055 individual days between 1992 and 2003, i.e. from near the maximum of cycle 22 to the declining phase of cycle 23 (top panel). The middle and lower panels show enlargements of four shorter intervals at different activity levels. The times corresponding to these zoom-ins are marked in the top panel by the double headed arrows under the roman numerals.

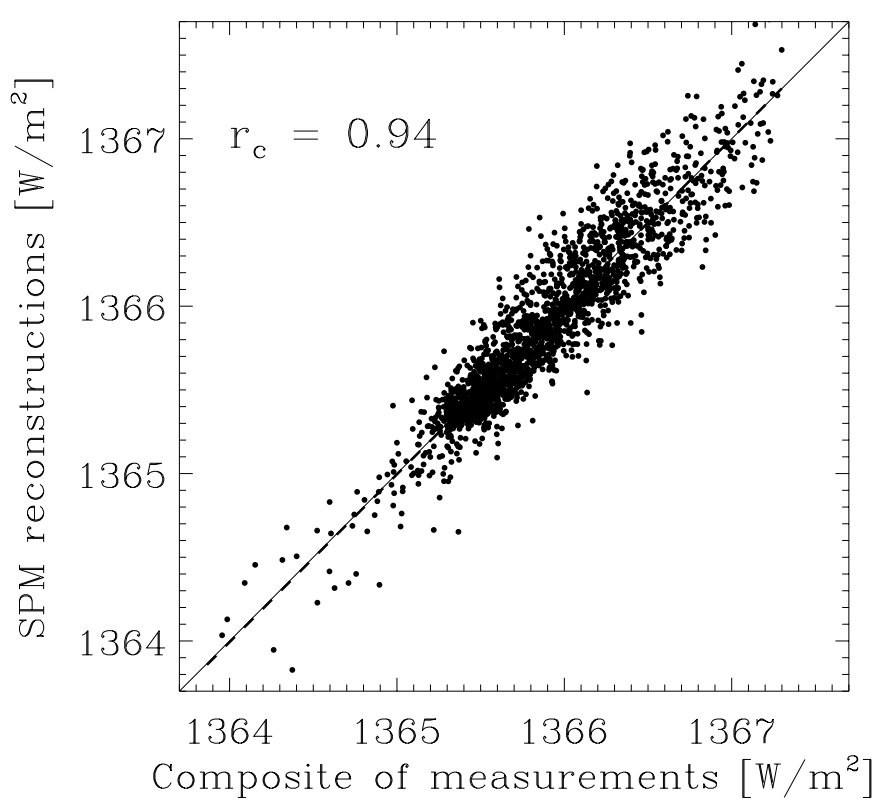

Fig. 2. Modelled total solar irradiance based on SPM data vs. composite of measurements. The correlation coefficient, $r_{\mathrm{c}}$, is indicated. The solid diagonal line represents the expectation values for a perfect model fit, a regression (dashed line) is hardly distinguishable from it.

The resulting rms deviation of the residual between the daily modelled and measured TSI is about $0.182 \mathrm{~W} \mathrm{~m}^{-2}$. The irradiance itself fluctuates by up to $4 \mathrm{~W} \mathrm{~m}^{-2}$, as can be easily seen from Fig. 2. The scatter in Fig. 3 may appear surprisingly large given the good agreement seen in Fig. 1. There is, however, no discrepancy and in the 4 lower panels of Fig. 1 we have not chosen periods for which the correspondence is particulary good between model and data. In fact, individual points showing large deviations between model and data can be seen in each of the four lower panels in Fig. 1. In order to illustrate that the chosen periods are typical, the intervals they cover have been marked in the top panel of Fig. 3 and the daily deviations are plotted for these chosen subintervals of time in the bottom four panels of Fig. 3. Here the filled circles represent daily differences.

Figure 3 confirms that we have no bias between cycles 22 and 23 , with the difference between reconstructed and measured irradiance being (statistically) unchanged over the whole period. Furthermore, we have calculated the average differences for cycles 22 and 23 separately. The boundary between the cycles is defined by the official minimum of the sunspot cycle in October 1996 (ftp://ftp.ngdc.noaa.gov). The value of the difference averaged over cycle 22 , i.e. between November 1992 and September 1996, is $0.006 \mathrm{~W} \mathrm{~m}^{-2}$. The corresponding value for cycle 23, i.e. between October 1996 and September 2003, is $-0.006 \mathrm{~W} \mathrm{~m}^{-2}$. We see, contrary to the findings of de Toma et al. (2001), but in agreement with those of de Toma et al. (2004), no difference in the behavior of cycles 22 and 23.

The correlation coefficients between the reconstructed and the measured irradiance (composite measurements) during cycles 22 (1992-1996) and 23 (1996-2003) and the slopes of the linear regressions (similar to Fig. 2) are listed in Table 1. 


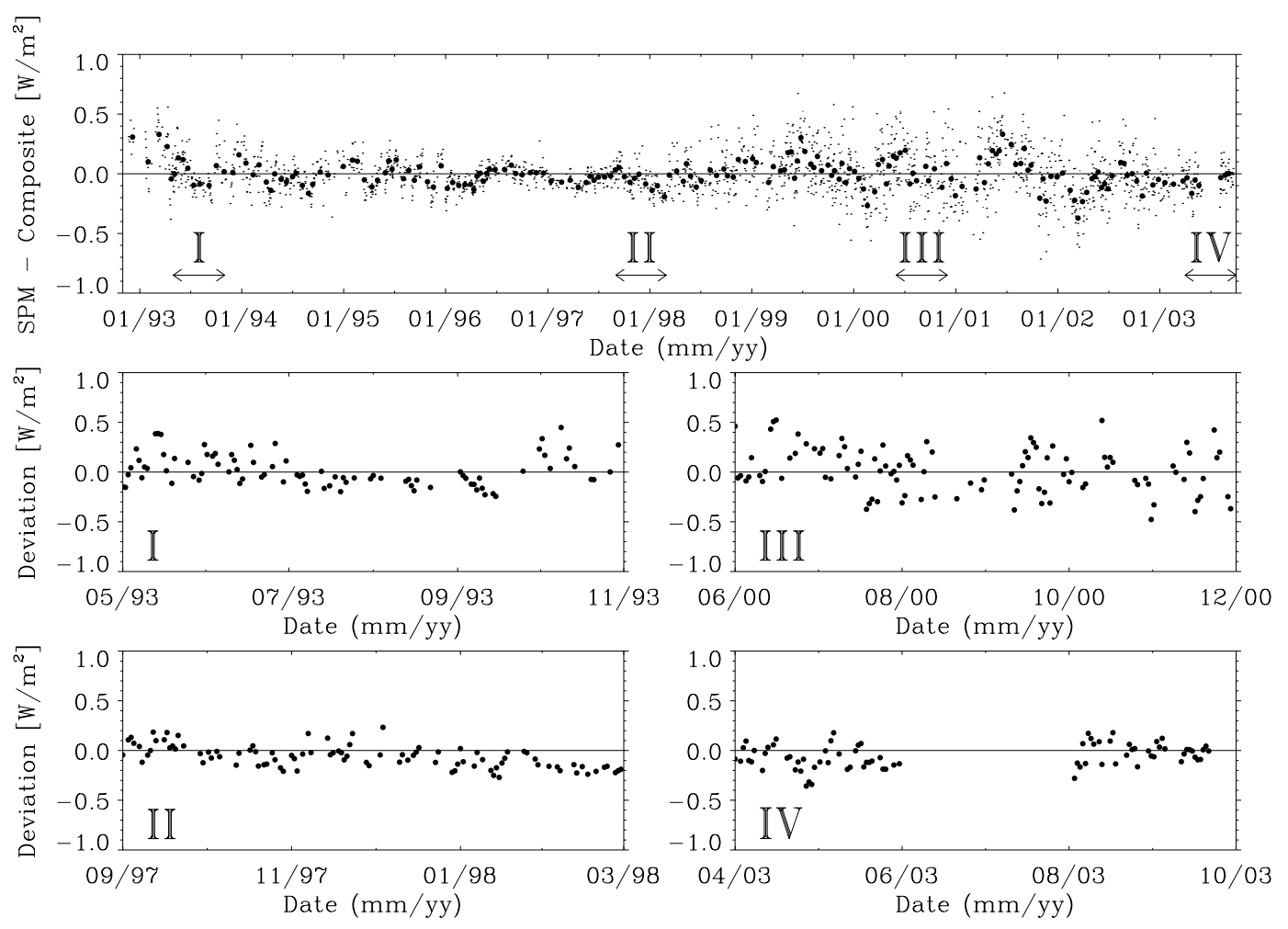

Fig. 3. Difference between the reconstructed total solar irradiance based on SPM data and the composite measurements. Each dot represents a daily value, the bigger filled circles 10 day averages. The solid line indicates difference $=0$ (top panel). The middle and lower panels show the same enlargements of four shorter intervals at different activity levels as in Fig. 1. The times corresponding to these zoom-ins are marked in the top panel by the double headed arrows under the roman numerals. Each filled circle now represents a daily value.

Table 1. Correlation coefficients, $r_{\mathrm{c}}$, and slopes of the linear fits to the scatter plots of modelled total solar irradiance based on SPM data vs. composite and ACRIM II measurements for different time periods.

\begin{tabular}{cccc}
\hline \hline Comparison & Time period & $r_{\mathrm{c}}$ & Slope \\
\hline Reconstr. vs. composite & $1992-2003$ & 0.94 & $1.003 \pm 0.080$ \\
Reconstr. vs. composite & $1992-1996$ & 0.86 & $0.932 \pm 0.022$ \\
Reconstr. vs. composite & $1996-2003$ & 0.93 & $1.018 \pm 0.011$ \\
Reconstr. vs. ACRIM II & $1992-2001$ & 0.84 & $1.006 \pm 0.017$ \\
Reconstr. vs. ACRIM II & $1992-1996$ & 0.78 & $1.049 \pm 0.034$ \\
Reconstr. vs. ACRIM II & $1996-2001$ & 0.81 & $0.958 \pm 0.023$ \\
ACRIM II vs. VIRGO & $1996-2001$ & 0.89 & $1.046 \pm 0.013$ \\
\hline
\end{tabular}

We find that the correlation coefficient is lower for cycle 22 than for cycle 23 as well as for the full period of time considered. Since Fig. 3 reveals no longer term trend, any such difference must be present at time-scales shorter than the solar rotation period. Irradiance changes at these time-scales are dominated by sunspots, which are responsible for the lowest measured TSI values and thus contribute significantly to fixing the slope of the regression line.

We recall that we have used composite measurements, which for the period considered are based on two different data sets, namely data from VIRGO and ACRIM II. To test this we have repeated the analysis, but now with only ACRIM II measurements. We determined $B_{\text {sat }}$ using the ACRIM II data alone for this reconstruction, obtaining $B_{\text {sat }}=230 \mathrm{G}$ for the whole period of time for which SPM and ACRIM II data are available (1992-2001). The resulting correlation coefficients and slopes are also listed in Table 1. The correlation coefficients and slopes are similar to each other for all three periods, although the slopes differ by more than the formal errors. The correlation coefficients obtained when comparing the reconstructions with ACRIM II are significantly lower than with VIRGO. We have also listed the correlation coefficient and slope between ACRIM II and VIRGO data in Table 1. This shows that the difference between instruments is currently of the same order as the difference between reconstruction and measurement. The difference between data from different radiometers can have a number of causes, see the discussion by, e.g. Fröhlich \& Lean (1998) and Fröhlich (2000).

We stress again that for the reconstructed total solar irradiance over all intervals we use the same value of the free parameter $B_{\text {sat }}$. Therefore, this analysis confirms that a single reconstruction can reproduce data from both cycles equally well. Given the difference between the two employed irradiance data sets, it is, however, not surprising that different $B_{\text {sat }}$ values are obtained when fitting ACRIM and composite data.

\section{Conclusions}

We have compared reconstructed TSI values based on SPM (spectromagnetograph on Kitt Peak) data with the composite 
TSI data set from PMOD/WRC taken between 1992 and 2003, and with the TSI data from ACRIM II taken between 1992 and 2001. The calculated total irradiance shows good agreement with observations on both short (days to months) and long (solar cycle) time-scales. We find that our model, based on the assumption that the solar irradiance changes are entirely caused by the evolution of the solar surface magnetic fields, can reproduce simultaneously the irradiance in both cycles 22 and 23. We find no sign of a bias between the reconstructed and measured total solar irradiance changes in cycles 22 and 23 on longer time-scales. Since our model is based on the assumption that irradiance changes on the considered time-scales (days to decades) are caused by the evolution of surface magnetic fields, its success lends further support to the validity of this assumption also beyond cycle 23 . However, we do find systematic differences between the behaviour of ACRIM II and VIRGO, which lead to seeming inconsistencies when considering irradiance composites.

Acknowledgements. We thank M. Fligge, D. M. Fluri and Y. C. Unruh for helpful discussions. The work of T. Wenzler was carried out under Poly project "Variability of the Sun and Global Climate" at ETH Zurich. The excellent working conditions and the generous support are herewith gratefully acknowledged.

\section{References}

de Toma, G., White, O. R., Chapman, G. A., et al. 2001, ApJ, 549, L131

de Toma, G., White, O. R., Chapman, G. A., et al. 2004, ApJ, 609, 1140

Fligge, M., Solanki, S. K., Meunier, N., \& Unruh, Y. C. 2000a, in The Solar Cycle and Terrestrial Climate, ed. A. Wilson, ESA Publ. SP-463, 117

Fligge, M., Solanki, S. K., \& Unruh, Y. C. 2000b, A\&A, 353, 380

Fröhlich, C. 2000, Space Sci. Rev., 94, 15

Fröhlich, C. 2003, in Solar Variability as an Input to the Earth's Environment, ed. A. Wilson, ESA Publ. SP-535, 183

Fröhlich, C., \& Lean, J. 1998, Geophys. Res. Lett., 25, 4377

Fröhlich, C., Romero, J., Roth, H., et al. 1995, Sol. Phys., 162, 101

Jones, H. P., Duvall, Jr., T. L., Harvey, J. W., et al. 1992, Sol. Phys., 139, 211

Krivova, N. A., Solanki, S. K., Fligge, M., \& Unruh, Y. C. 2003, A\&A, 399, L1

Unruh, Y. C., Solanki, S. K., \& Fligge, M. 1999, A\&A, 345, 635

Wenzler, T., Solanki, S. K., Krivova, N. A., \& Fluri, D. M. 2004, A\&A, 427, 1031

Willson, R. C. 1994, in The Sun as a Variable Star, ed. J. M. Pap, C. Fröhlich, H. S. Hudson, \& S. K. Solanki (Cambridge: Cambridge Univ. Press), IAU Coll., 143, 54 